\title{
Modeling and Simulation of High Operating Temperature MWIR Photo Detector Based on Mercury Cadmium Telluride
}

\author{
Mohammad Nadimi and Ali Sadr
}

\begin{abstract}
High operating temperatures infrared photo detectors are needed for improving the performance of these systems. To obtain high device performance at higher temperatures, the thermally generated noise required to be reduced. Minority-carrier extraction and exclusion techniques are the approaches for decreasing the thermal noise of infrared systems. In the present work, an $\mathrm{Hg1-xCdxTe}$ photodiode was studied and simulated for operation in the MWIR region. A simulation of the device was carried out by using ATLAS software from SILVACO ${ }^{\circledR}$ in order to study the performance of the photo detector at near room temperatures. The device was characterized in respect of energy band diagram, carrier concentration, doping profile, dark current and responsively.
\end{abstract}

Index Terms-HgCdTe, infrared photo detector, simulation.

\section{INTRODUCTION}

Recent progress of infrared technology, in the field of thermal imaging, remote sensing, astronomy, etc., has been made due to the successful development of high performance infrared detectors over the last five decades [1]. Many materials were investigated in the infrared field. Mercury Cadmium Telluride $(\mathrm{HgCdTe})$ is one of the most prominent semiconductor material for mid $(2-5$ um $)$ and long-wavelength (8-12 um) infrared photon detectors. This is because of the fact that the mole fraction $(\mathrm{x})$ of cadmium in $\mathrm{Hgl-xCdxTe}$ can be adjusted suitably to tailor the energy band-gap of the material to match the wavelength of the above atmospheric windows [2]. However it was assumed previously that the desirable properties of these infrared photon detectors are accessible only at cryogenic temperatures. The requirement for cryogenic cooling systems adds a significant amount of cost, power consumption and weight to the infrared detectors [3]. There is, therefore, a great interest in achieving higher operating temperature infrared systems. The limiting factor for cooling requirements was the dark current in the detector devices [4]. The dark current in $\mathrm{HgCdTe}(\mathrm{MCT})$ infrared detectors was limited by Auger processes at near-room temperatures of operation. One of the promising approaches to achieving higher operating temperature infrared photon detectors was first proposed by Ashley et al. [5] which was based on

Manuscript received March5 2011; revised July 11, 2011.

Mohammad Nadimi is with the Department of Electrical Engineering, Iran University of Science and Technology, Tehran, Iran. (nadimi@ymail.com).

Ali Sadr is with the Department of Electrical Engineering, Iran University of Science and Technology, Tehran, Iran. (sadr@iust.ac.ir). minority-carrier extraction and exclusion techniques under non-equilibrium condition as described later.

Steady-state numerical simulations of $\mathrm{HgCdTe}$ Auger-suppressed infrared photodiodes by using a Sentaurus Device, a commercial software package by Synopsis, were reported by Emilie et al. [6-8]. In this paper, a comprehensive model of device was designed and simulated using ATLAS device software from SILVACO ${ }^{\circledR}$ for electrical characterization of the non-equilibrium MCT photo detectors.

\section{Simulation}

A two-dimensional numerical simulation of an $\mathrm{HgCdTe}$ infrared detector was carried out using ATLAS software. A program was developed separately in DECKBUILD window interfaced with ATLAS for calculating different characteristics of the photo detector. The simulation included the solution of five decoupled equations using Newton iteration method. Carrier and doping densities were calculated using Fermi-Dirac statistics. In calculation of mobility the concentration dependent model was used and for extracting the dark characteristics, the optical, SRH and Auger recombination processes were taken into account. Different recombination rates are given as:

$$
\begin{aligned}
& \mathrm{R}_{\mathrm{np}}^{\mathrm{OPT}}=\mathrm{C}_{\mathrm{C}}^{\mathrm{OPT}}\left(\mathrm{pn}-\mathrm{n}_{\mathrm{i}}^{2}\right) \\
& \mathrm{R}_{\mathrm{SRH}}=\frac{\mathrm{pn}-\mathrm{n}_{\mathrm{i}}^{2}}{\mathrm{~T}_{\mathrm{po}}\left\{\mathrm{n}+\mathrm{n}_{\mathrm{i}} \exp \left(\mathrm{E}_{\mathrm{t}} / \mathrm{KT}\right)\right\}+\mathrm{T}_{\mathrm{no}}\left\{\mathrm{p}+\mathrm{n}_{\mathrm{i}} \exp \left(-\mathrm{E}_{\mathrm{t}} / \mathrm{KT}\right)\right\}} \\
& \mathrm{R}_{\text {Auger }}=\mathrm{C}_{\mathrm{n}}\left(\mathrm{pn}^{2}-\mathrm{nn}_{\mathrm{i}}^{2}\right)+\mathrm{C}_{\mathrm{p}}\left(\mathrm{p}^{2} \mathrm{n}-\mathrm{pn}_{\mathrm{i}}^{2}\right)
\end{aligned}
$$

where $\mathrm{C}_{\mathrm{OPT}}$ is capture rate of carriers, $\mathrm{Cn}$ and $\mathrm{Cp}$ are Auger coefficients for electrons and holes respectively, $\mathrm{n}$ and $\mathrm{p}$ are equilibrium electron and hole concentration, Et is energy level of trap, ni is intrinsic carrier concentration and Tpo and Tno are SRH lifetime of holes and electrons respectively [9-11].

\section{DeVICE Description}

The performance of MCT infrared detectors was limited by Auger processes at near-room temperatures of operation. The use of non-equilibrium techniques based on the minority carrier phenomena of exclusion and extraction were proposed to raise the operating temperature of infrared detectors.

The goal of these techniques was to reduce the electron and hole concentrations from their intrinsic levels to the 
values typical of extrinsic conditions so that the control of devices was regained by the do pants, and the leakage currents and resulting noise in devices were decreased to the acceptable levels by suppressing the Auger mechanisms [12].

The structure of the device consisted of a central very lowly doped ' $v$ ' (n-type) or ' $\pi$ ' (p-type) active layer where the infrared radiation was to be absorbed and two heavily doped $\mathrm{n}+$ and $\mathrm{p}+$ larger-band-gap regions yielded a three-layer $n+-v-p+$ structure (for n-type active layer). Under reverse bias and at temperatures where the middle region was near intrinsic, minority carrier extraction occurred at the diode junction $(v-\mathrm{p}+)$ resulting in large reductions in the minority carrier densities in its vicinity. The $n+$ material formed an excluding contact to the $v$ material, thus preventing the injection of carriers from replacing those removed at the diode junction, so the hole density dropped by several orders of magnitude throughout the $v$ region. The electron concentration also fell down, to keep space charge neutrality, from the equilibrium, intrinsic level to the extrinsic value set by the doping level. The net decrease in the carrier density below its equilibrium value resulted in a drastic reduction in the dark current enabling the device to operate at higher temperatures [12-13].

\section{RESULTS AND DISCUSSIONS}

In this work, ATLAS software was used to simulate the electrical properties of the MCT photo detectors by using appropriate material parameters given in Table 1 [14]. Fig. 1 displays the structure of the MWIR detector and its corresponding band diagram. The net doping profile of the photodiode is shown in Fig. 2.

TABLE 1- MATERIAL PARAMETER OF HGL-XCDXTE

\begin{tabular}{|c|c|}
\hline parameter & value \\
\hline$E_{g}$ & $\begin{array}{c}0.302+1.93 x-0.81 x^{2}+0.832 x^{3}+5.354 \times 10^{-4} \\
\times(1-2 x) T\end{array}$ \\
\hline $\boldsymbol{m}_{e}$ & $\left\lceil-0.6+\left.6.333\left(\frac{2}{E_{g}}+\frac{1}{E_{g}+1}\right)\right|^{-1} m_{0}\right.$ \\
\hline $\boldsymbol{m}_{p}$ & $0.55 m_{0}$ \\
\hline $\boldsymbol{u}_{e}$ & $9 \times 10^{4}\left\lceil\frac{0.2}{x}\right\rceil^{7.5} \mathrm{~T}^{-0.2\left(\frac{0.2}{x}\right)^{0.6}}$ \\
\hline $\boldsymbol{u}_{h}$ & $0.01 u_{e}$ \\
\hline $\boldsymbol{\varepsilon}$ & $20.5-15.5 x+5.7 x^{2}$ \\
\hline$\chi$ & $4.23-0.813\left(E_{g}-0.0083\right)$ \\
\hline
\end{tabular}

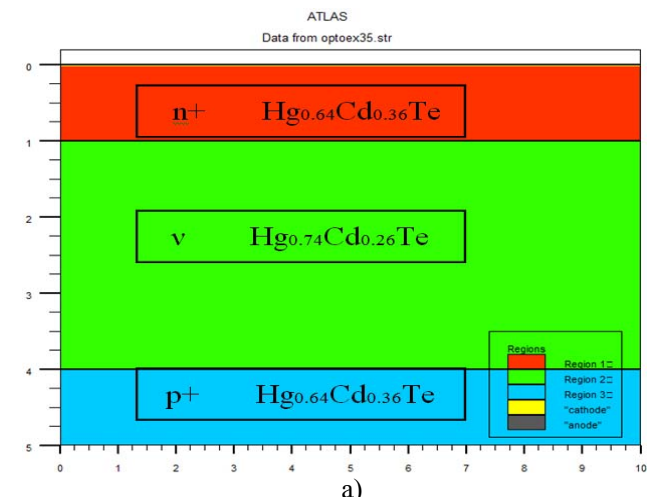

a)

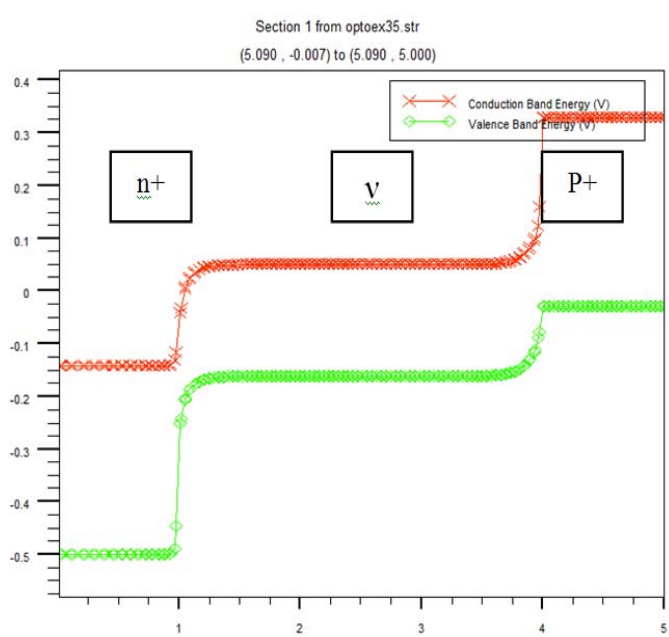

b)

Fig. 1. Structure of the MWIR detector and its corresponding band diagram.

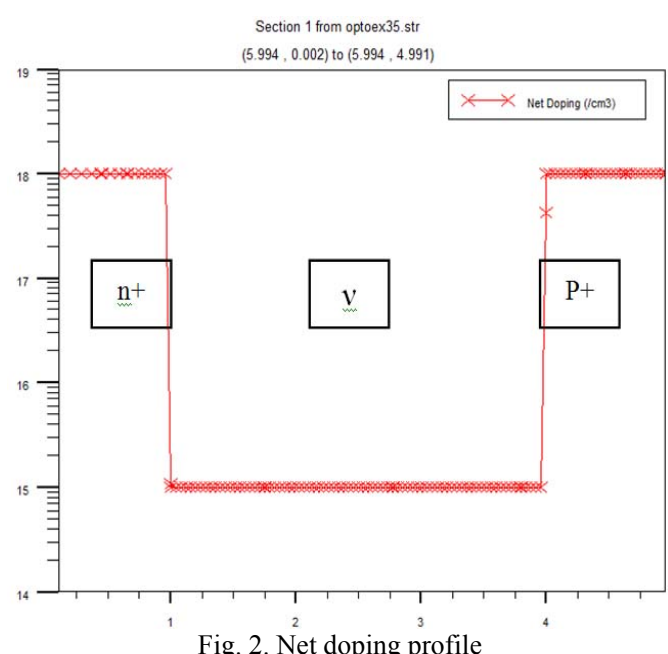

Fig. 3 shows the electron and hole concentration profiles of the $n+-v-p+$ heterojunction diode at equilibrium and under reverse-bias conditions. By applying sufficient reverse bias inside the $v$ region the hole density dropped by several orders of magnitude and the electron concentration also felled down to the doping level (as discussed in section III). Fig. 4 displays the simulated dark I-V characteristics of the photo detector at $295 \mathrm{k}$.

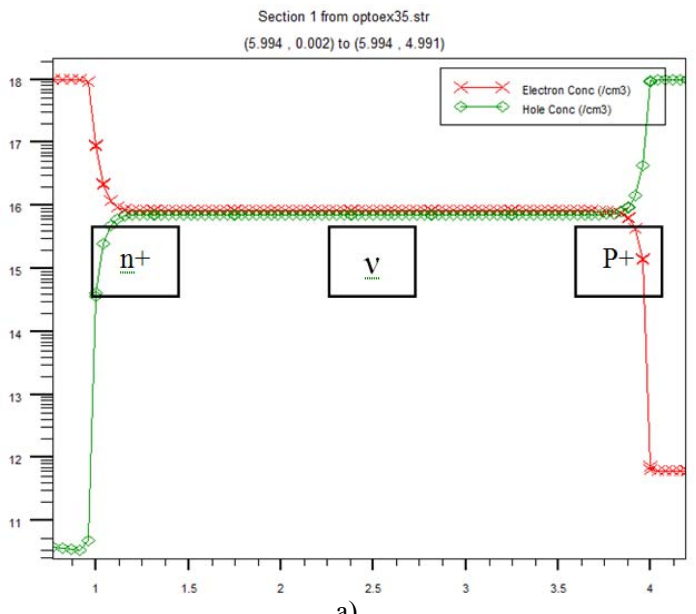




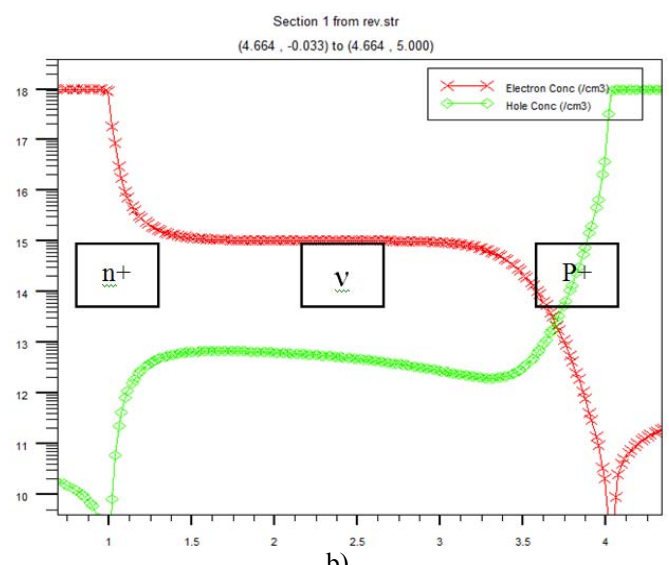

b)

Fig. 3. Carrier concentrations :(a) Equilibrium, and (b) bias $=-0.35$

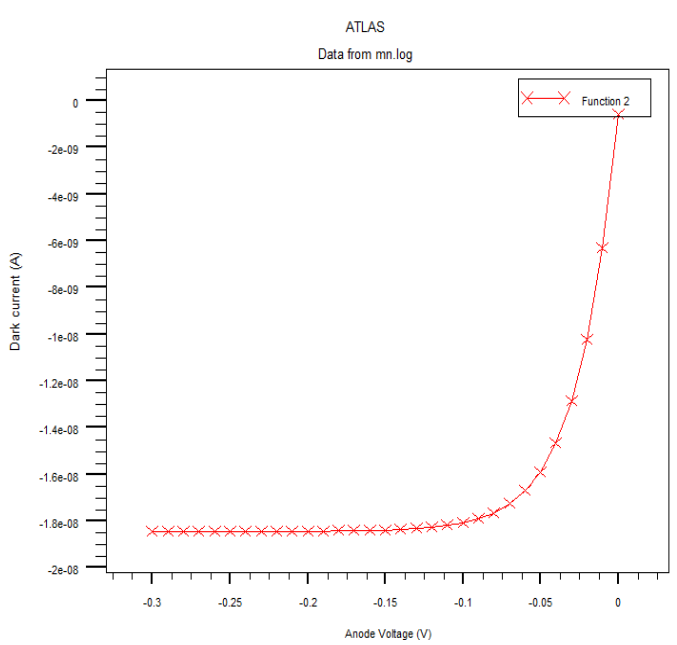

Fig. 4. Variation of dark current with reverse voltage

Fig. 5 shows the variation of responsivity of the MWIR photodetector with wavelength of operation. It can be seen from this figure that the current responsivity increased with wavelength of operation and had a peak value at $\lambda=5 \mathrm{um}$.

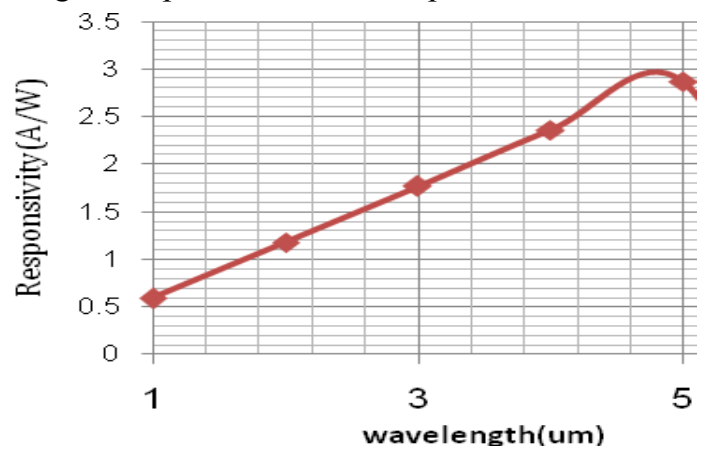

Fig. 5. Variation of responsivity with wavelength.

\section{CONCLUSION}

In this paper, no equilibrium operations of $\mathrm{HgCdTe}$ photo detector were studied. Modeling and Simulation were performed by using ATLAS simulator. The energy band diagram, carrier concentration, doping profile, dark current and responsively obtained by using ATLAS simulator were presented. The simulation and modeling described in this work can be used for optimizing the existing infrared detectors and developing new devices.

\section{REFERENCES}

[1] A. Rogalski, "heterostructure infrared photodiodes," Semiconductor Physics, Quantum Electronics \& Optoelectronics, vol. 3, no. 2, pp. 111-120, 2000.

[2] P. K. Saxena and P. Chakrabarti, "Computer modeling of MWIR single heterojunction photodetector based on mercury cadmium telluride," Infrared Physics \&Technology, vol. 52, pp. 196-203, 2009.

[3] A. Piotrowski, P. Madejczyk, W. Gawron, K. Kłos, J. Pawluczyk, M. Grudzień, J. Piotrowski, and A. Rogalski, "MOCVD HgCdTe heterostructures for uncooled infrared," Proc. SPIE, vol. 5732, pp. 273-284, 2005.

[4] S. Velicu, C. H. Grein, P.Y. Emelie, A. Itsuno, J.D. Phillips, and P.S. Wijewarnasuriya, "MWIR and LWIR HgCdTe infrared detectors operated with reduced cooling requirements," J. Electron. Mater, vol. 39, no. 7, pp.873-881, 2010.

[5] T. Ashley and C. T. Elliot, "Nonequilibrium devices for infrared detection," Electron. Lett, vol. 21, no. 10, pp. 451-452, 1985.

[6] P.Y. Emelie, J.D. Phillips, S. Velicu, and P.S. Wijewarnasuriya, "Parameter extraction of $\mathrm{HgCdTe}$ infrared photodiodes exhibiting Auger suppression,” J. Phys D: Appl. Phys, vol. 42, pp. 234003, 2009.

[7] P.Y. Emelie, S. Velicu, C. H. Grein, J.D. Phillips, P.S Wijewarnasuriya, and N. K. Dhar, "Modeling of LWIR HgCdTe Auger-Suppressed Infrared Photodiodes under Nonequilibrium Operation,” J. Electron. Mater, vol. 37, no. 9, pp.1362-1368, 2008.

[8] P.Y. Emelie, J.D. Phillips, S. Velicu, and C. H. Grein, "modeling and design considerations of $\mathrm{HgCdTe}$ infrared photodiodes under nonequilibrium operation," J. Electron. Mater, vol. 36, no. 8, pp. 846-852, 2007.

[9] A.D.D. Dwivedi, A. Mittal, A. Agrawal, P. Chakrabarti, “Analytical modeling and simulation of $\mathrm{N}+-\mathrm{InP} / \mathrm{n} 0$-In0.53Ga0.47As/p+-In0.53Ga0.47As p-i-n photodetector for optical fiber communication," Infrared Physics \&Technology, vol. 53, pp. 236-245, 2010

[10] M. Nadimi, A. sadr, " Simulation of Uncooled InSb Infrared Detectors ", in Proc. IEEE, Int. Conf. on Modeling, Simulation and Control (ICMSC 2010), Cairo, Egypt,pp. 504-507, 2010.

[11] M. Nadimi, A. sadr, " Computer Modeling of MWIR Homojunction Photodetector based on Indium Antimonide", in Proc. IEEE, Int. Conf. on Manufacturing Science and Technology (ICMST 2010), Kuala Lumpur, Malaysia, pp.373-376, 2010.

[12] T. Ashley and C. T. Elliott, "Operation and properties of narrow-gap semiconductor devices near room temperature using non-equilibrium techniques," Semicond. Sci. Technol, vol. 6, pp. c99-c105, 1991.

[13] R. Ashokan, T. S. Lee, J. Zhao, Y. Chang, and S. Sivananthan, "Mercury Cadmium Telluride For High Operating Temperature Infrared Detectors," Proceedings for the Army Science Conference, 2004.

[14] ATLAS User's Manual, Device Simulation Software, SILVACO International, Santa Clara, CA 95054, 20 